\title{
Highly Stretchable and Transparent Microfluidic Strain Sensors for Monitoring Human Body Motions
}

\section{- Supporting Information -}

\author{
Sun Geun Yoon ${ }^{1}$, Hyung-Jun Koo ${ }^{2}$, and Suk Tai Chang ${ }^{1 *}$ \\ ${ }^{1}$ School of Chemical Engineering and Materials Science, Chung-Ang University, Seoul 156- \\ 756, Republic of Korea \\ E-mail: stchang@cau.ac.kr \\ ${ }^{2}$ Department of Chemical and Biomolecular Engineering, Seoul National University of \\ Science and Technology, Seoul 139-743, Republic of Korea
}


Table S1. Comparison of the degree of hysteresis (DH) of electrical response between the strain sensors based on conductive solid nanomaterials and the microfluidic strain sensors used in this study.

\begin{tabular}{|c|c|c|c|}
\hline & Materials & Tensile Strain (\%) & Degree of Hysteresis (\%) \\
\hline Muth et al. [Ref. 3] & Carbon grease ink & 100 & 9.04 \\
\hline Li et al. [Ref. 6] & Graphene mesh & 8 & 4.74 \\
\hline Yamada et al. [Ref. 9] & Carbon nanotubes & 200 & 71.38 \\
\hline Jeong et al. [Ref. 10] & Graphene foam & 0.8 & 6.06 \\
\hline Amjadi et al. [Ref. 12] & Silver nanowires & 60 & 37.81 \\
\hline Yao et al. [Ref. 13] & Silver nanowires & 50 & 5.57 \\
\hline \multirow[t]{3}{*}{ Lee et al. [Ref. 17] } & Silver nanoparticles & 200 & 20.4 \\
\hline & & $200\left(2.9 \mathrm{~mm} \mathrm{~s}^{-1}\right)$ & 2.41 \\
\hline & & $25\left(2.9 \mathrm{~mm} \mathrm{~s}^{-1}\right)$ & 3.73 \\
\hline \multirow[t]{3}{*}{ This work } & Ionic Liquids & $25\left(4.9 \mathrm{~mm} \mathrm{~s}^{-1}\right)$ & 1.89 \\
\hline & & $25\left(9.35 \mathrm{~mm} \mathrm{~s}^{-1}\right)$ & 37.44 \\
\hline & & $25\left(13.58 \mathrm{~mm} \mathrm{~s}^{-1}\right)$ & 51.57 \\
\hline
\end{tabular}



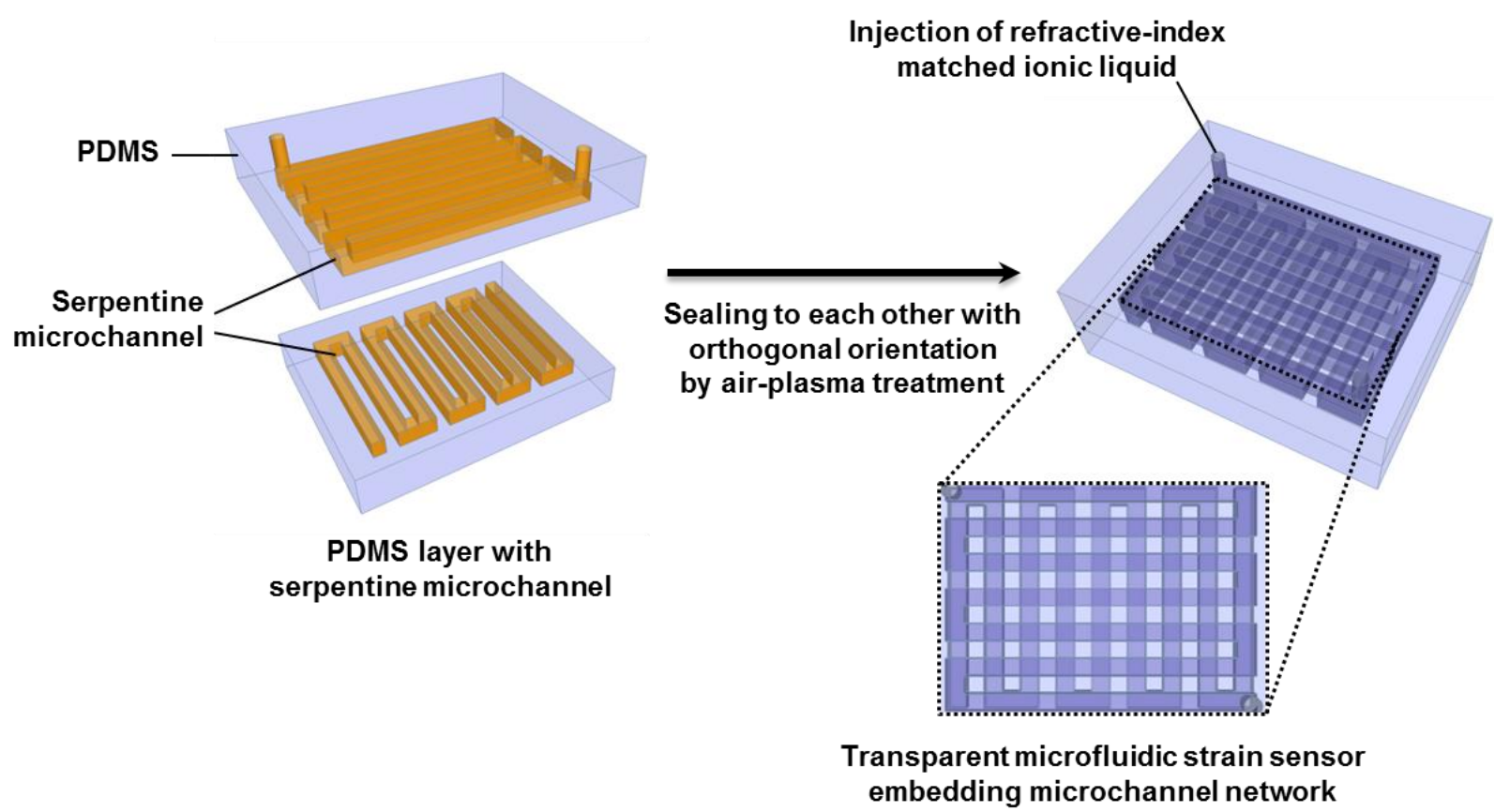

Figure S1. Schematic illustration of fabrication process for optically transparent microfluidic strain sensors embedded with microfluidic networks. 

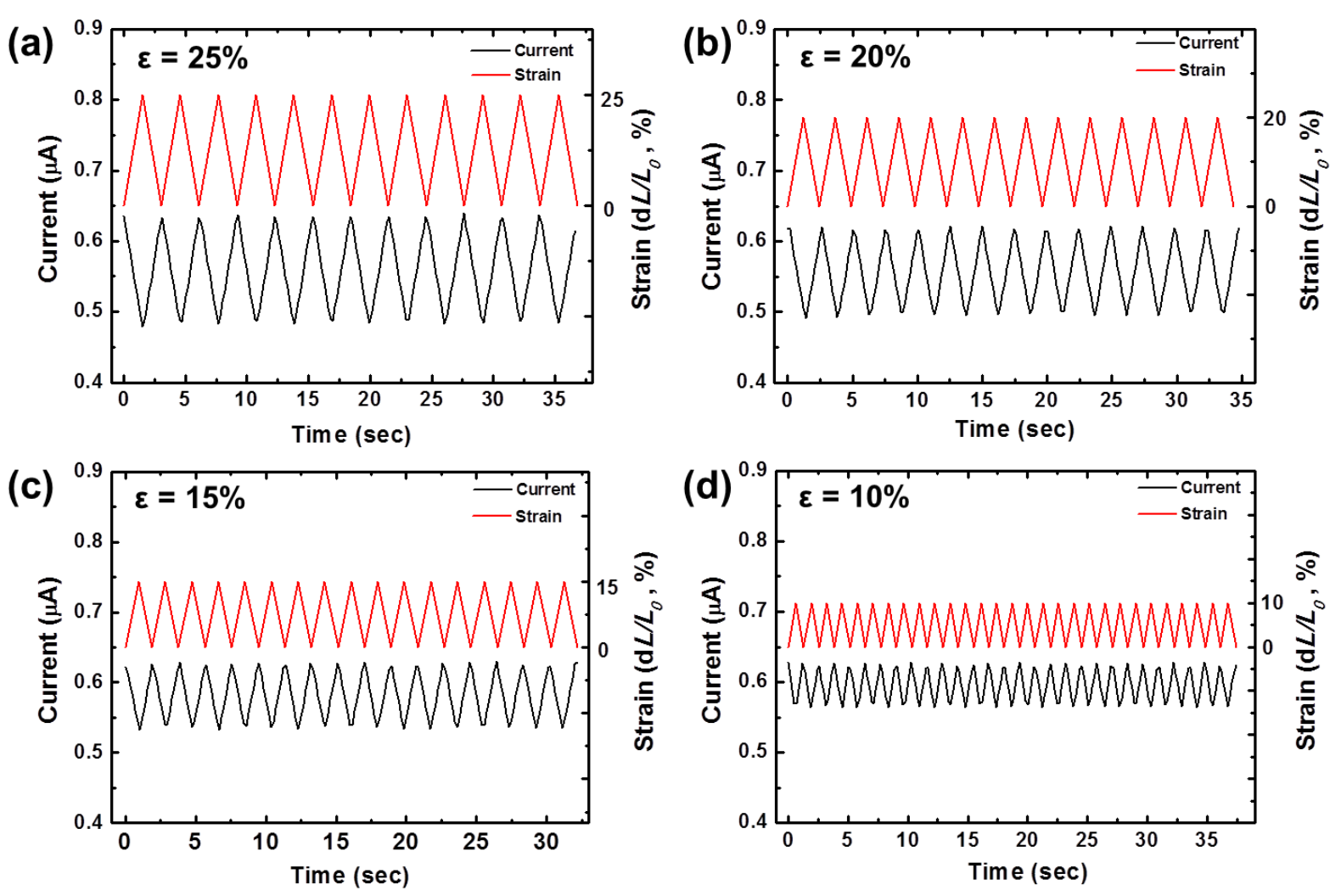

Figure S2. Current response and applied strain variation as a function of time for the PDMS microfluidic strain sensor subjected to cyclic deformation with various tensile strains at a speed of $4.9 \mathrm{~mm} \mathrm{~s}^{-1}$. (a) $\varepsilon=25 \%$, (b) $\varepsilon=20 \%$, (c) $\varepsilon=15 \%$, (d) $\varepsilon=10 \%$. 

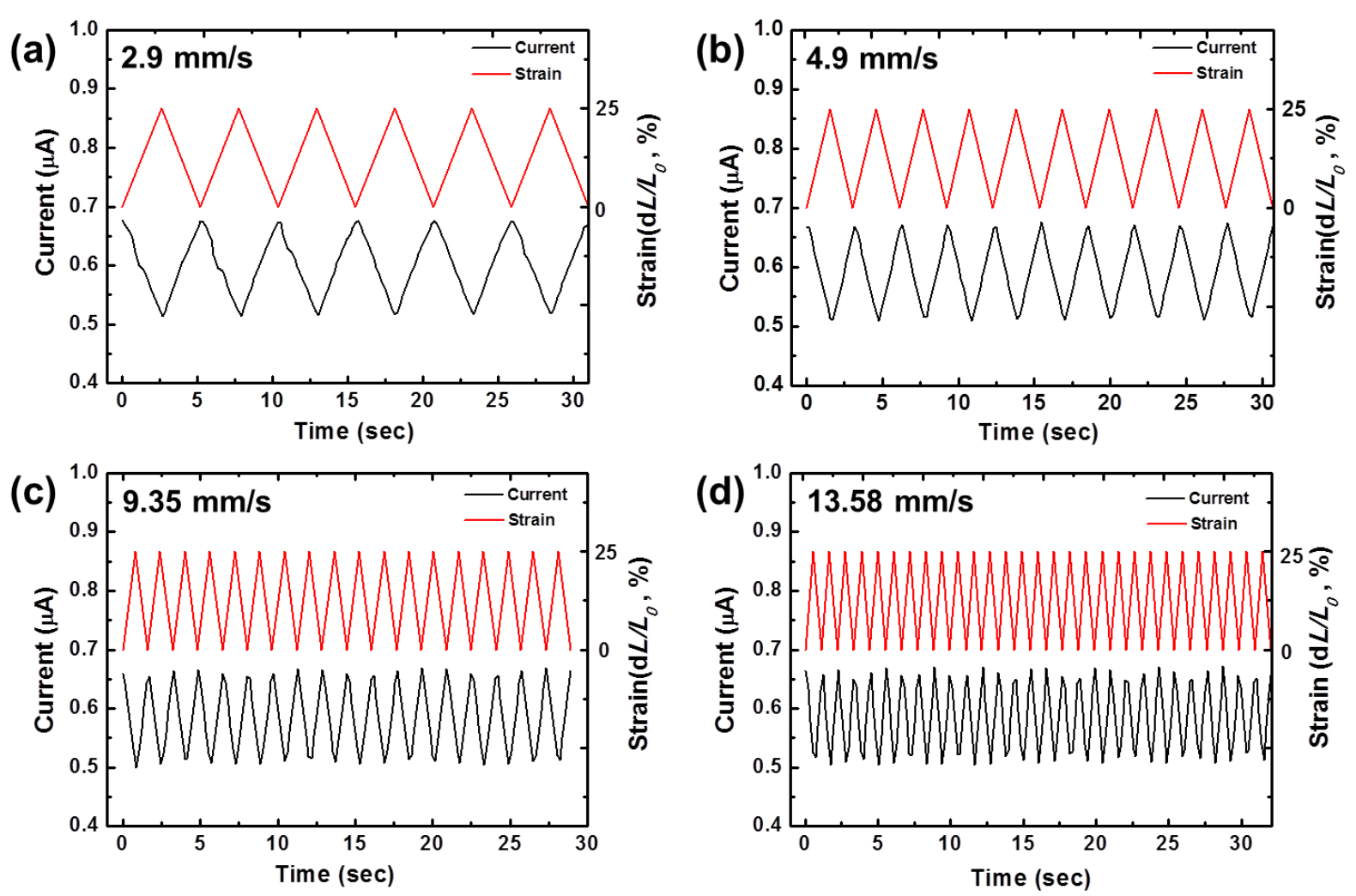

Figure S3. Current response and applied strain variation as a function of time for the PDMS microfluidic strain sensor subjected to cyclic deformation up to $25 \%$ tensile strain at various strain speeds. (a) $2.9 \mathrm{~mm} \mathrm{~s}^{-1}$, (b) $4.9 \mathrm{~mm} \mathrm{~s}^{-1}$, (c) $9.35 \mathrm{~mm} \mathrm{~s}^{-1}$, (d) $13.58 \mathrm{~mm} \mathrm{~s}^{-1}$.

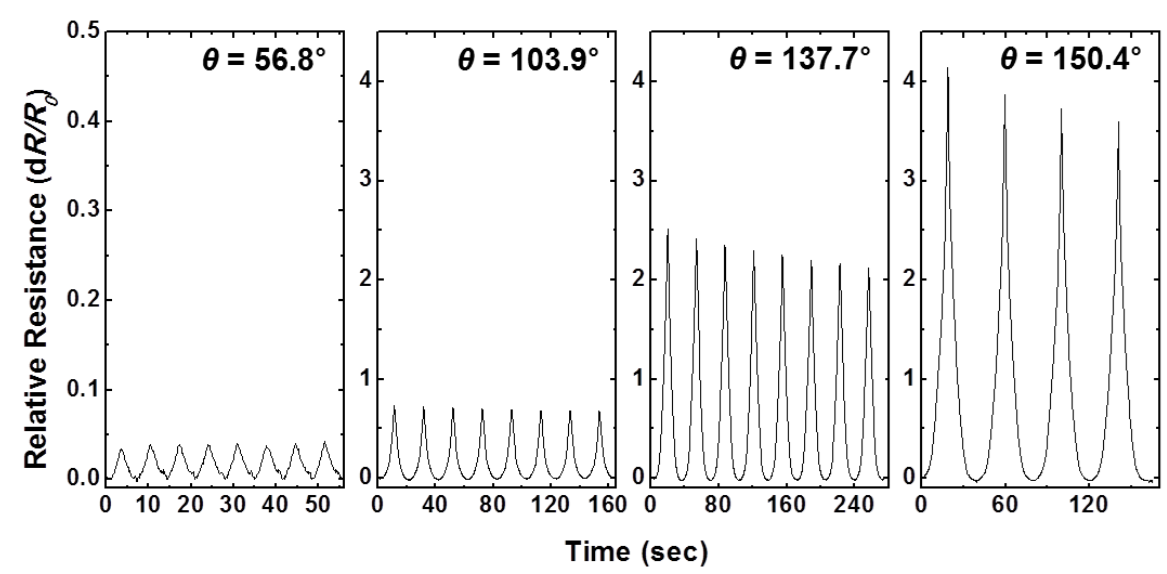

Figure S4. Relative resistance change as a function of time for the microfluidic strain sensor subjected to cyclic bending deformation up to different maximum bending angles at a speed of $2.9 \mathrm{~mm} \mathrm{~s}^{-1}$. 


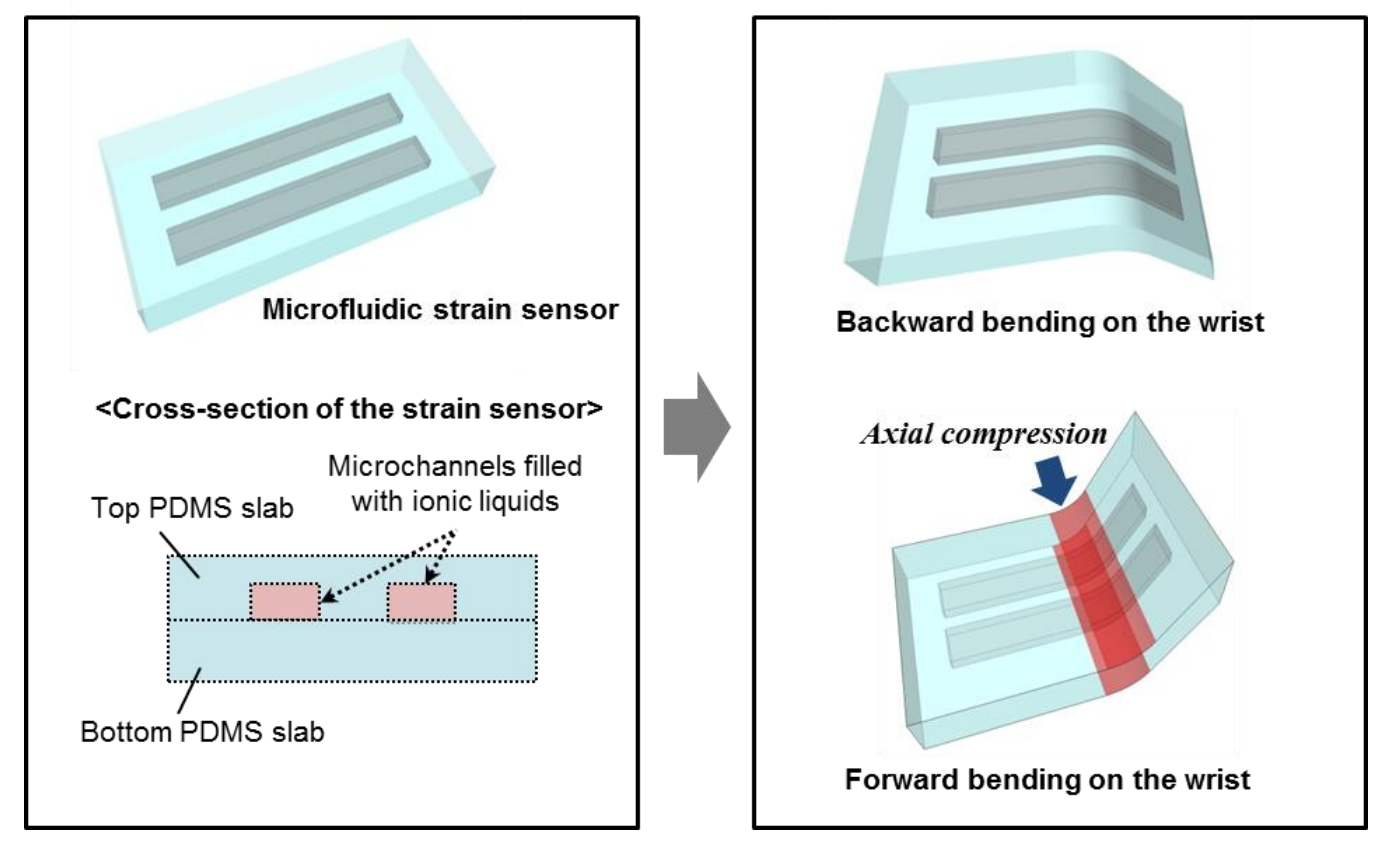

Figure S5. Schematic illustrations of the microfluidic strain sensor used in measuring the motions of a wrist. Since two microchannels in this strain sensor are embedded in the top PDMS layer, during the bending motion of the wrist toward the top layer (defined as forward bending mode) with a small angle, the axial compression occurs in the microchannels. Thus, this compression could have caused the decrease of electrical resistance across the ionic liquids filled in the channels, as shown in Figure $5 \mathrm{~b}$. 